\title{
Traveling waves for a diffusive SIR model with delay and nonlinear incidence
}

\author{
Yanmei Wanga,b, Guirong Liua,*, Aimin Zhao ${ }^{\mathrm{a}}$ \\ a School of Mathematical Sciences, Shanxi University, Taiyuan, Shanxi 030006, China. \\ ${ }^{b}$ School of Applied Mathematics, Shanxi University of Finance and Economics, Taiyuan, Shanxi 030006, China.
}

Communicated by R. Saadati

\begin{abstract}
This paper is concerned with the existence and non-existence of traveling wave solutions for a diffusive SIR model with delay and nonlinear incidence. First, we construct a pair of upper and lower solutions and a bounded cone. Then we prove the existence of traveling wave by using Schauder's fixed point theorem and constructing a suitable Lyapunov functional. The nonexistence of traveling wave is obtained by two-sided Laplace transform. Moreover, numerical simulations support the theoretical results. Finally, we also obtain that the minimal wave speed is decreasing with respect to the latent period and increasing with respect to the diffusion rate of infected individuals.
\end{abstract}

Keywords: SIR model, traveling wave, time delay, nonlinear incidence.

2010 MSC: 35K57, 35C07, 92D30.

(C)2018 All rights reserved.

\section{Introduction}

The SIR epidemic models and their various extensions have been frequently used in the study of theoretical epidemiology. It is well known that the disease propagation is relevant to not only the time variation, but also the space variation which is sufficient to describe a disease's diffusion and plays an important role in the disease spreading. In this paper, we consider traveling waves of the following diffusive SIR model with delay and nonlinear incidence

$$
\left\{\begin{array}{l}
S_{t}(x, t)=d_{1} S_{x x}(x, t)+B_{1}-\mu S(x, t)-F(S(x, t)) G(I(x, t-\tau)), \\
I_{t}(x, t)=d_{2} I_{x x}(x, t)+F(S(x, t)) G(I(x, t-\tau))-(\mu+\gamma) I(x, t), \\
R_{t}(x, t)=d_{3} R_{x x}(x, t)+\gamma I(x, t)-\mu R(x, t),
\end{array}\right.
$$

where $S(x, t), I(x, t), R(x, t)$ denote the total number of susceptible, infected, and removed individuals, respectively, in the time-space coordinate $(t, x)$, and $d_{i}>0(i=1,2,3)$ are their diffusion rates. The

\footnotetext{
*Corresponding author

Email address: lgr5791@sxu.edu.cn (Guirong Liu)
}

doi: $10.22436 /$ jnsa.011.12.03

Received: 2017-12-12 Revised: 2018-08-04 Accepted: 2018-08-23 
parameter $B_{1}>0$ represents the entering flux of the susceptible; $\mu>0$ is the natural death rate; $\gamma>0$ is the recovery rate of the infective population; and $\tau>0$ is the latent period of the disease.

For the original system of SIR model, it is assumed that the transmission is instantaneous, and does not consider the spatial variation (see [7]). In order to be more realistic, the spatial variation should be considered. There have been many works on spatial epidemic models (see $[3,15,16])$. For instance, Hosono and Ilyas [5] investigated the existence and nonexistence of traveling wave solutions for the simple diffusive epidemic model

$$
\left\{\begin{array}{l}
S_{t}(x, t)=d_{1} S_{x x}(x, t)-\beta S(x, t) I(x, t) \\
I_{t}(x, t)=d_{2} I_{x x}(x, t)+\beta S(x, t) I(x, t)-\gamma I(x, t)
\end{array}\right.
$$

Here, bilinear incidence $\beta$ SI is considered. However, the disease transmission process may have a nonlinear incidence rate (see [6, 8-10]). Korobeinikov and Maini [10] considered the incidence of the form $f(S) g(I)$. In addition, in epidemiological models, delay can be caused by many factors. So the influences of delays should be considered when modeling epidemic process (see $[1,2,11-14,17,18])$. Based on these modeling mechanism, Bai and $\mathrm{Wu}[1]$ studied the following diffusive SIR model with delay and nonlinear incidence rate

$$
\left\{\begin{array}{l}
S_{t}(x, t)=d_{1} S_{x x}(x, t)-F(S(x, t)) G(I(x, t-\tau)), \\
I_{t}(x, t)=d_{2} I_{x x}(x, t)+F(S(x, t)) G(I(x, t-\tau))-\gamma I(x, t), \\
R_{t}(x, t)=d_{3} R_{x x}(x, t)+\gamma I(x, t),
\end{array}\right.
$$

where the incidence rate is the nonlinear incidence rate $F(S) G(I)$. By using Schauder's fixed theorem, the existence and nonexistence of traveling wave solutions are obtained. Moreover, the existence of $S(+\infty)$ is established. However, the author does not get the exact value of $S(+\infty)$. In order to be more practical, we consider an epidemic model of birth and death rates. Fu [4] studied the following diffusive SIR model with delay and saturated incidence rate

$$
\left\{\begin{array}{l}
S_{t}(x, t)=d_{1} S_{x x}(x, t)+\mu(\Lambda-S(x, t))-\frac{\beta S(x, t) I(x, t-\tau)}{1+\alpha I(x, t-\tau)}, \\
I_{t}(x, t)=d_{2} I_{x x}(x, t)+\frac{\beta S(x, t) I(x, t-\tau)}{1+\alpha I(x, t-\tau)}-(\mu+\gamma) I(x, t), \\
R_{t}(x, t)=d_{3} R_{x x}(x, t)+\gamma I(x, t)-\mu R(x, t)
\end{array}\right.
$$

where the constant $\mu \Lambda$ is the recruitment rate of the susceptible population, the incidence rate is saturated incidence rate $\frac{\beta S I}{1+\alpha \mathrm{I}}$. Using the upper and lower solutions and the Schauder's fixed point theorem, the existence of traveling waves is obtained.

Motivated the above works, we shall consider traveling waves of system (1.1).

For simplicity, let

$$
\widetilde{S}(x, t)=\frac{\mu}{B_{1}} S(x, t), \quad \widetilde{I}(x, t)=\frac{\mu}{B_{1}} I(x, t), \quad \widetilde{R}(x, t)=\frac{\mu}{B_{1}} R(x, t),
$$

and

$$
f(u)=\frac{\mu}{B_{1}} F\left(\frac{B_{1}}{\mu} u\right), g(u)=G\left(\frac{B_{1}}{\mu} u\right) .
$$

By dropping the tilde for convenience, we then consider the following system

$$
\left\{\begin{array}{l}
S_{t}(x, t)=d_{1} S_{x x}(x, t)+\mu(1-S(x, t))-f(S(x, t)) g(I(x, t-\tau)), \\
I_{t}(x, t)=d_{2} I_{x x}(x, t)+f(S(x, t)) g(I(x, t-\tau))-(\mu+\gamma) I(x, t), \\
R_{t}(x, t)=d_{3} R_{x x}(x, t)+\gamma I(x, t)-\mu R(x, t) .
\end{array}\right.
$$

Throughout this paper, the following assumptions hold.

$\left(\mathrm{A}_{1}\right) \mathrm{f} \in \mathrm{C}([0, \infty),[0, \infty)), f(0)=0 ; f^{\prime}(u)>0$ for any $u \geqslant 0 ; f^{\prime}(u)$ is bounded on $[0, \infty)$;

$\left(A_{2}\right) g \in C([0, \infty),[0, \infty)), g(0)=0 ; g^{\prime}(u)>0$ and $g^{\prime \prime}(u) \leqslant 0$ for any $u \geqslant 0$. 
Since the first two equations of (1.2) form a closed system, we only consider the following system

$$
\left\{\begin{array}{l}
S_{t}(x, t)=d_{1} S_{x x}(x, t)+\mu(1-S(x, t))-f(S(x, t)) g(I(x, t-\tau)) \\
I_{t}(x, t)=d_{2} I_{x x}(x, t)+f(S(x, t)) g(I(x, t-\tau))-(\mu+\gamma) I(x, t)
\end{array}\right.
$$

It is easy to see that (1.3) always has a disease-free equilibrium $(1,0)$. Furthermore, if $R_{0}:=\frac{f(1) g^{\prime}(0)}{\gamma+\mu}>1$, there exists a unique positive endemic equilibrium $\left(s^{*}, i^{*}\right)$ satisfying $0<s^{*}<1$ and $0<i^{*}<\frac{\mu}{\mu+\gamma}$ (see Lemma A.1 in Appendix). If $R_{0}<1$, there exists no positive endemic equilibrium. By a traveling wave solution of system (1.3), we mean a solution of system (1.3) of the form

$$
S(x, t)=s(\xi), \quad I(x, t)=i(\xi), \quad \xi=x+c t \in R,
$$

with the boundary condition

$$
(s, i)(-\infty)=(1,0) \text { and }(s, i)(+\infty)=\left(s^{*}, i^{*}\right),
$$

where $c>0$ is a constant. In order to show the existence of traveling wave, in addition to $R_{0}>1$, we give the following assumption.

$\left(\mathrm{A}_{3}\right)$ There exists $\mathrm{B}>0$ such that $\mathrm{g}(\mathrm{B})=\frac{\mu+\gamma}{\mathrm{f}(1)} \mathrm{B}$.

It is easy to see that $i^{*}<B$. That is, $i^{*}<\min \left\{\frac{\mu}{\mu+\gamma}, B\right\}$. Then for any $\xi \in R$, substituting $(s(\xi), i(\xi))$ into (1.3) yields

$$
\left\{\begin{array}{l}
\operatorname{cs}^{\prime}(\xi)=d_{1} s^{\prime \prime}(\xi)+\mu(1-s(\xi))-f(s(\xi)) g(i(\xi-c \tau)) \\
\operatorname{ci}^{\prime}(\xi)=d_{2} i^{\prime \prime}(\xi)+f(s(\xi)) g(i(\xi-c \tau))-(\mu+\gamma) \mathfrak{i}(\xi)
\end{array}\right.
$$

To obtain the existence of traveling wave solutions of (1.3), we only need to get the existence of solutions for system (1.5) satisfying (1.4). Our main theorems are stated as follows.

Theorem 1.1. If $\left(\mathrm{A}_{1}\right)-\left(\mathrm{A}_{3}\right)$ hold and $\mathrm{R}_{0}>1$, then for any $\mathrm{c}>\mathrm{c}^{*}$, system (1.5) admits a nontrivial and nonnegative solution $(\mathrm{s}(\xi), i(\xi))$ satisfying the following properties:

(i) $0<s(\xi)<1$ and $0<i(\xi)<B$ for all $\xi \in R$;

(ii) $\lim _{\xi \rightarrow-\infty}(s(\xi), i(\xi))=(1,0), \lim _{\xi \rightarrow+\infty}(s(\xi), i(\xi))=\left(s^{*}, i^{*}\right)$, i.e., system (1.3) exists a traveling wave solution with speed c;

(iii) $\lim _{\xi \rightarrow-\infty} i(\xi) e^{-\lambda_{1} \xi}=1$ and $\lim _{\xi \rightarrow-\infty}\left(s^{\prime}(\xi), i^{\prime}(\xi)\right)=\lim _{\xi \rightarrow+\infty}\left(s^{\prime}(\xi), i^{\prime}(\xi)\right)=(0,0)$,

where $\mathrm{c}^{*}$ and $\lambda_{1}$ are shown in Lemma 2.1.

Theorem 1.2. Assume $\left(\mathrm{A}_{1}\right)$ and $\left(\mathrm{A}_{2}\right)$ hold. One of the following conditions holds:

(i) $R_{0}=1$ and system (1.5) exists a unique positive endemic equilibrium $\left(\mathrm{s}^{*}, i^{*}\right)$;

(ii) $\mathrm{R}_{0}>1$ and $\mathrm{c} \in\left(0, \mathrm{c}^{*}\right)$.

Then system (1.5) does not exist nontrivial and nonnegative solution satisfying (1.4).

This paper is organized as follows. In Section 2, we first construct a pair of upper and lower solutions for system (1.5). Then, we construct a bounded cone and verify the conditions of Schauder's fixed point theorem. In Section 3, the existence of solutions for system (1.5) is proved. Moreover, we obtain that the solution satisfies the boundary conditions $(s, i)(+\infty)=\left(s^{*}, i^{*}\right)$ by constructing a Lyapunov functional. In addition, we establish the non-existence of solutions for system (1.5) satisfying (1.4) by two-sided Laplace transform. In Section 4, some numerical simulations are introduced to demonstrate the analytical results. In Section 5, we give a brief discussion. Finally, some auxiliary lemma is given in Appendix. 


\section{Preliminary}

Linearizing the second equation of $(1.5)$ around the point $(1,0)$ yields

$$
d_{2} i^{\prime \prime}(\xi)-c i^{\prime}(\xi)+f(1) g^{\prime}(0) i(\xi-c \tau)-(\mu+\gamma) \mathfrak{i}(\xi)=0,
$$

whose characteristic equation is given by

$$
\Delta(\lambda, c):=d_{2} \lambda^{2}-c \lambda+f(1) g^{\prime}(0) e^{-\lambda c \tau}-(\mu+\gamma)=0 .
$$

Then we have the following result.

Lemma 2.1. Suppose $\mathrm{R}_{0}>1$. Then there exists $\mathrm{c}^{*}>0$ and $\lambda^{*}>0$ such that

$$
\left.\frac{\partial \Delta(\lambda, \mathrm{c})}{\partial \lambda}\right|_{\left(\lambda^{*}, \mathrm{c}^{*}\right)}=0 \text { and } \Delta\left(\lambda^{*}, \mathrm{c}^{*}\right)=0
$$

Moreover, $\Delta(\lambda, c)$ also satisfies:

(i) if $\mathrm{c}>\mathrm{c}^{*}$, then $\Delta(\lambda, \mathrm{c})=0$ has two positive roots $\lambda_{1}=\lambda_{1}$ (c) and $\lambda_{2}=\lambda_{2}$ (c) with $0<\lambda_{1}(\mathrm{c})<\lambda^{*}<\lambda_{2}$ (c) $<$ $\infty$, and $\Delta(\lambda, c)<0$ for $\lambda \in\left(\lambda_{1}(\mathrm{c}), \lambda_{2}(\mathrm{c})\right)$ and $\Delta(\lambda, \mathrm{c})>0$ for $\lambda \in\left(0, \lambda_{1}(\mathrm{c})\right) \cup\left(\lambda_{2}(\mathrm{c}),+\infty\right)$;

(ii) if $0<\mathrm{c}<\mathrm{c}^{*}$, then $\Delta(\lambda, \mathrm{c})>0$ for all $\lambda \in[0,+\infty)$.

Proof. It follows from $R_{0}>1$ that $\Delta(0, c)=f(1) g^{\prime}(0)-\mu-\gamma>0$. For each fixed $c>0,\left.\frac{\partial \Delta(\lambda, c)}{\partial \lambda}\right|_{\lambda=0}=$ $-c-c \tau f(1) g^{\prime}(0)<0$, and $\frac{\partial^{2} \Delta(\lambda, c)}{\partial \lambda^{2}}>0$ for any $\lambda \in R$.

Since $\Delta(\lambda, 0)=d_{2} \lambda^{2}+f(1) g^{\prime}(0)-\mu-\gamma>0, \frac{\partial \Delta(\lambda, c)}{\partial c}<0$ for all $\lambda>0$, and for any fixed $\lambda>0$, $\Delta(\lambda, \mathrm{c})<0$ if $\mathrm{c}$ is sufficiently large. Define

$$
c^{*}:=\sup \{c>0 \mid \Delta(\lambda, c)>0, \forall \lambda \in R\} .
$$

It is easy to see that $c^{*} \in(0, \infty)$ and $\Delta\left(\lambda, c^{*}\right)=0$ has a unique root denoted by $\lambda^{*}$. We can easily prove that $\lambda^{*}>0$, and $\Delta\left(\lambda^{*}, \mathrm{c}^{*}\right)=0,\left.\frac{\partial \Delta(\lambda, \mathrm{c})}{\partial \lambda}\right|_{\left(\lambda^{*}, \mathrm{c}^{*}\right)}=0$. Then, by a simple discussion, we can obtain the conclusions of (i) and (ii).

In the following, we always assume $R_{0}>1$ and $c>c^{*}$. Now we define four nonnegative continuous functions $s_{+}, s_{-}, i_{+}$, and $i_{-}$as follows:

$$
\begin{aligned}
& s_{+}(\xi)=1, \\
& i_{+}(\xi)= \begin{cases}e^{\lambda_{1} \xi}, & \text { if } \xi<\xi_{0}, \\
B, & \text { if } \xi \geqslant \xi_{0},\end{cases} \\
& s_{-}(\xi)= \begin{cases}1-\sigma e^{\alpha \xi}, & \text { if } \xi<\xi_{1}, \\
0, & \text { if } \xi \geqslant \xi_{1},\end{cases} \\
& i_{-}(\xi)= \begin{cases}e^{\lambda_{1} \xi}\left(1-M e^{\eta \xi}\right), & \text { if } \xi<\xi_{2}, \\
0, & \text { if } \xi \geqslant \xi_{2},\end{cases}
\end{aligned}
$$

where $\sigma, \alpha, \eta, M$ are all positive constants satisfying $0<\eta<\min \left\{\lambda_{2}-\lambda_{1}, \lambda_{1}\right\}, 0<\alpha<\lambda_{1}, \sigma>1, M>1$ and

$$
\xi_{0}=\frac{1}{\lambda_{1}} \ln B, \quad \xi_{1}=\frac{1}{\alpha} \ln \frac{1}{\sigma}<\xi_{0}, \quad \xi_{2}=\frac{1}{\eta} \ln \frac{1}{M}<\xi_{1} .
$$

Definition 2.2. $\left(s_{+}, i_{+}\right)$and $\left(s_{-}, i_{-}\right)$are called a pair of upper and lower solutions of (1.5), if $s_{+}, i_{+}, s_{-}, i_{-}$ are almost everywhere continuously differentiable and satisfy

$$
\left\{\begin{array}{l}
c s_{+}^{\prime}(\xi) \geqslant d_{1} s_{+}^{\prime \prime}(\xi)+\mu\left(1-s_{+}(\xi)\right)-f\left(s_{+}(\xi)\right) g\left(i_{-}(\xi-c \tau)\right), \\
c s_{-}^{\prime}(\xi) \leqslant d_{1} s_{-}^{\prime \prime}(\xi)+\mu\left(1-s_{-}(\xi)\right)-f\left(s_{-}(\xi)\right) g\left(i_{+}(\xi-c \tau)\right), \\
c i_{+}^{\prime}(\xi) \geqslant d_{2} i_{+}^{\prime \prime}(\xi)+f\left(s_{+}(\xi)\right) g\left(i_{+}(\xi-c \tau)\right)-(\mu+\gamma) i_{+}(\xi), \\
c i_{-}^{\prime}(\xi) \leqslant d_{2} i_{-}^{\prime \prime}(\xi)+f\left(s_{-}(\xi)\right) g\left(i_{-}(\xi-c \tau)\right)-(\mu+\gamma) i_{-}(\xi) .
\end{array}\right.
$$


It is obvious that $s_{+}(\xi)$ satisfies the inequality

$$
c s_{+}^{\prime}(\xi) \geqslant d_{1} s_{+}^{\prime \prime}(\xi)+\mu\left(1-s_{+}(\xi)\right)-f\left(s_{+}(\xi)\right) g\left(i_{-}(\xi-c \tau)\right), \quad \xi \in R .
$$

Lemma 2.3. The function $i_{+}(\xi)$ satisfies the inequality

$$
c i_{+}^{\prime}(\xi) \geqslant d_{2} i_{+}^{\prime \prime}(\xi)+f\left(s_{+}(\xi)\right) g\left(i_{+}(\xi-c \tau)\right)-(\mu+\gamma) i_{+}(\xi)
$$

for all $\xi \neq \xi_{0}$.

Proof. For $\xi>\xi_{0}$, since $i_{+}(\xi)=B$ in $\left(\xi_{0}, \infty\right),\left(A_{2}\right)$, and $\left(A_{3}\right)$, the inequality (2.2) holds. For $\xi<\xi_{0}$, it follows from Lemma 2.1 and $g\left(i_{+}\right) \leqslant g^{\prime}(0) i_{+}$that

$$
c i_{+}^{\prime}(\xi)=d_{2} i_{+}^{\prime \prime}(\xi)+f(1) g^{\prime}(0) i_{+}(\xi-c \tau)-(\mu+\gamma) i_{+}(\xi), \quad \xi \in R,
$$

which yields (2.2).

Lemma 2.4. Assume that $0<\alpha<\lambda_{1}$ is sufficiently small. Then

$$
c s_{-}^{\prime}(\xi) \leqslant d_{1} s_{-}^{\prime \prime}(\xi)+\mu\left(1-s_{-}(\xi)\right)-f\left(s_{-}(\xi)\right) g\left(i_{+}(\xi-c \tau)\right)
$$

for any $\xi \neq \xi_{1}$, and $\sigma>1$ is sufficiently large.

Proof. If $\xi>\xi_{1}$, then $s_{-}(\xi)=0$. Further, it is easy to see that (2.3) holds. If $\xi<\xi_{1}$, then $s_{-}(\xi)=$ $1-\sigma e^{\alpha \xi} \in(0,1)$. This, together with $\left(A_{1}\right)$, yields $f\left(s_{-}(\xi)\right)<f(1)$. We only need to prove that

$$
-c \alpha \sigma e^{\alpha \xi}+d_{1} \alpha^{2} \sigma e^{\alpha \xi}+f(1) g\left(i_{+}(\xi-c \tau)\right)-\mu \sigma e^{\alpha \xi} \leqslant 0 .
$$

Note that $\alpha \in\left(0, \lambda_{1}\right)$ is sufficiently small, and $\sigma>1$ is large enough. Hence

$$
-c \alpha \sigma+d_{1} \alpha^{2} \sigma+f(1) g^{\prime}(0) \sigma^{\frac{\alpha-\lambda_{1}}{\alpha}} e^{-\lambda_{1} c \tau}-\mu \sigma \leqslant 0,
$$

which implies

$$
\left.-c \alpha \sigma e^{\alpha \xi}+d_{1} \alpha^{2} \sigma e^{\alpha \xi}+f(1) g^{\prime}(0) i_{+}(\xi-c \tau)\right)-\mu \sigma e^{\alpha \xi} \leqslant 0, \quad \xi<\xi_{1} .
$$

In addition,

$$
g\left(i_{+}(\xi-c \tau)\right) \leqslant g^{\prime}(0) i_{+}(\xi-c \tau), \quad \xi<\xi_{1} .
$$

This, together with (2.5), yields the inequality (2.4) holds.

Lemma 2.5. Suppose that $0<\eta<\min \left\{\lambda_{2}-\lambda_{1}, \lambda_{1}\right\}$. Then

$$
c i_{-}^{\prime}(\xi) \leqslant d_{2} i_{-}^{\prime \prime}(\xi)+f\left(s_{-}(\xi)\right) g\left(i_{-}(\xi-c \tau)\right)-(\mu+\gamma) i_{-}(\xi)
$$

for large enough $M>1$, and for any $\xi \neq \xi_{2}$.

Proof. The proof is similar to that of [1, Lemma 2.4]. Hence we omit its proof.

From (2.1) and Lemmas 2.3-2.5, we obtain that $\left(s_{+}, i_{+}\right)$and $\left(s_{-}, i_{-}\right)$are a pair of upper and lower solutions of (1.5). In addition, $s_{-}(\xi)<s_{+}(\xi), i_{-}(\xi)<i_{+}(\xi)$ for any $\xi \in R$.

Let $\Lambda_{i}^{-}, \Lambda_{i}^{+}(i=1,2)$ be the roots of $d_{i} \Lambda^{2}-c \Lambda-\alpha_{i}=0$, where $\alpha_{1}, \alpha_{2}$ are all positive constants satisfying

$$
\alpha_{1}>\mu+\sup _{0 \leqslant s \leqslant 1}\left\{f^{\prime}(s)\right\} g^{\prime}(0) B, \quad \alpha_{2}>\mu+\gamma .
$$


Further,

$$
\Lambda_{i}^{-}=\frac{c-\sqrt{c^{2}+4 d_{i} \alpha_{i}}}{2 d_{i}}<0, \quad \Lambda_{i}^{+}=\frac{c+\sqrt{c^{2}+4 d_{i} \alpha_{i}}}{2 d_{i}}>0 .
$$

Let $\beta \in\left(0, \min \left\{-\Lambda_{1}^{-},-\Lambda_{2}^{-}\right\}\right)$be a constant. Denote

$$
\mathrm{B}_{\beta}\left(\mathrm{R}, \mathrm{R}^{2}\right):=\left\{(s, i) \in \mathrm{C}\left(\mathrm{R}, \mathrm{R}^{2}\right): \sup _{\xi \in \mathrm{R}}|s(\xi)| e^{-\beta|\xi|}<+\infty, \sup _{\xi \in R}|i(\xi)| e^{-\beta|\xi|}<+\infty\right\},
$$

with norm

$$
|(s, i)|_{\beta}:=\max \left\{\sup _{\xi \in R}|s(\xi)| e^{-\beta|\xi|}, \sup _{\xi \in R}|i(\xi)| e^{-\beta|\xi|}\right\} .
$$

It is easy to check that $\left(B_{\beta}\left(R, R^{2}\right),|\cdot|_{\beta}\right)$ is a Banach space. Denote

$$
\Gamma=\left\{(s, i) \in B_{\beta}\left(R, R^{2}\right) \mid s_{-}(\xi) \leqslant s(\xi) \leqslant s_{+}(\xi), i_{-}(\xi) \leqslant i(\xi) \leqslant i_{+}(\xi), \xi \in R\right\} .
$$

Clearly, $\left(s_{-}, i_{-}\right) \in \Gamma,\left(s_{+}, i_{+}\right) \in \Gamma ; \Gamma$ is bounded, closed, and convex in $B_{\beta}\left(R, R^{2}\right)$.

Next, for any $(s, i) \in \Gamma, \xi \in R$, we define an operator $F: \Gamma \rightarrow C\left(R, R^{2}\right)$ by

$$
F(s, i)(\xi)=\left(F_{1}(s, i)(\xi), F_{2}(s, i)(\xi)\right),
$$

where

$$
\begin{aligned}
F_{1}(s, i)(\xi)= & \rho_{1} \int_{-\infty}^{\xi} e^{\Lambda_{1}^{-}(\xi-x)}\left[\alpha_{1} s(x)-f(s(x)) g(i(x-c \tau))-\mu s(x)+\mu\right] d x \\
& +\rho_{1} \int_{\xi}^{\infty} e^{\Lambda_{1}^{+}(\xi-x)}\left[\alpha_{1} s(x)-f(s(x)) g(i(x-c \tau))-\mu s(x)+\mu\right] d x \\
F_{2}(s, i)(\xi)= & \rho_{2} \int_{-\infty}^{\xi} e^{\Lambda_{2}^{-(\xi-x)}\left[\alpha_{2} i(x)+f(s(x)) g(i(x-c \tau))-(\mu+\gamma) i(x)\right] d x} \\
& +\rho_{2} \int_{\xi}^{\infty} e^{\Lambda_{2}^{+}(\xi-x)}\left[\alpha_{2} i(x)+f(s(x)) g(i(x-c \tau))-(\mu+\gamma) i(x)\right] d x
\end{aligned}
$$

and

$$
\rho_{1}=\frac{1}{d_{1}\left(\Lambda_{1}^{+}-\Lambda_{1}^{-}\right)}, \quad \rho_{2}=\frac{1}{d_{2}\left(\Lambda_{2}^{+}-\Lambda_{2}^{-}\right)} .
$$

One can easily see that any fixed point of $F$ is a solution of (1.5). Hence the existence of solution for (1.5) is reduced to verify that the operator F satisfies the conditions of Schauder's fixed point theorem. Here, we have the following lemmas.

Lemma 2.6. The operator $F$ maps $\Gamma$ into $\Gamma$.

Proof. For any $(s, i) \in \Gamma, \xi \in R$, we have

$$
\begin{aligned}
F_{1}(s, i)(\xi) & \leqslant \rho_{1} \int_{-\infty}^{\xi} e^{\Lambda_{1}^{-}(\xi-x)}\left[\left(\alpha_{1}-\mu\right) s(x)+\mu\right] d x+\rho_{1} \int_{\xi}^{\infty} e^{\Lambda_{1}^{+}(\xi-x)}\left[\left(\alpha_{1}-\mu\right) s(x)+\mu\right] d x \\
& \leqslant \alpha_{1} \rho_{1}\left[\int_{-\infty}^{\xi} e^{\Lambda_{1}^{-}(\xi-x)} d x+\int_{\xi}^{\infty} e^{\Lambda_{1}^{+}(\xi-x)} d x\right]=\alpha_{1} \rho_{1}\left(\frac{1}{\Lambda_{1}^{+}}-\frac{1}{\Lambda_{1}^{-}}\right)=s_{+}(\xi) .
\end{aligned}
$$

In view of $\alpha_{1}>\mu+\sup _{0 \leqslant s \leqslant 1}\left\{f^{\prime}(s)\right\} g^{\prime}(0) B$, we obtain that $\left(\alpha_{1}-\mu\right) s-f(s) g(i)$ is increasing with respect to $s$.

By Lemma 2.4, for any $\xi \neq \xi_{1}$, we get

$$
c s_{-}^{\prime}(\xi) \leqslant d_{1} s_{-}^{\prime \prime}(\xi)+\mu\left(1-s_{-}(\xi)\right)-f\left(s_{-}(\xi)\right) g\left(i_{+}(\xi-c \tau)\right)
$$




$$
\begin{aligned}
& =d_{1} s_{-}^{\prime \prime}(\xi)+\mu-\alpha_{1} s_{-}(\xi)+\alpha_{1} s_{-}(\xi)-f\left(s_{-}(\xi)\right) g\left(i_{+}(\xi-c \tau)\right)-\mu s_{-}(\xi) \\
& \leqslant d_{1} s_{-}^{\prime \prime}(\xi)+\mu-\alpha_{1} s_{-}(\xi)+\alpha_{1} s(\xi)-f(s(\xi)) g(i(\xi-c \tau))-\mu s(\xi)
\end{aligned}
$$

that is,

$$
\alpha_{1} s(\xi)-f(s(\xi)) g(i(\xi-c \tau))-\mu s(\xi)+\mu \geqslant-d_{1} s_{-}^{\prime \prime}(\xi)+c s_{-}^{\prime}(\xi)+\alpha_{1} s_{-}(\xi) .
$$

This, together with the definition of $F_{1}$, yields

$$
\begin{aligned}
F_{1}(s, i)(\xi) \geqslant & \rho_{1} \int_{-\infty}^{\xi} e^{\Lambda_{1}^{-}(\xi-x)}\left[-d_{1} s_{-}^{\prime \prime}(x)+c s_{-}^{\prime}(x)+\alpha_{1} s_{-}(x)\right] d x \\
& +\rho_{1} \int_{\xi}^{\infty} e^{\Lambda_{1}^{+}(\xi-x)}\left[-d_{1} s_{-}^{\prime \prime}(x)+c s_{-}^{\prime}(x)+\alpha_{1} s_{-}(x)\right] d x \\
\geqslant & s_{-}(\xi)+\rho_{1} d_{1} \min \left\{e^{\Lambda_{1}^{+}\left(\xi-\xi_{1}\right)}, e^{\Lambda_{1}^{-}\left(\xi-\xi_{1}\right)}\right\}\left[s_{-}^{\prime}\left(\xi_{1}+0\right)-s_{-}^{\prime}\left(\xi_{1}-0\right)\right] \geqslant s_{-}(\xi)
\end{aligned}
$$

for any $\xi \neq \xi_{1}$.

Next, we prove $i_{-}(x) \leqslant F_{2}(s, i)(x) \leqslant i_{+}(x)$. It follows from (2.6) that the function $\left(\alpha_{2}-\mu-\gamma\right) i$ is increasing with respect to $i$. This implies $F_{2}(s, i)(\xi) \geqslant 0$ for any $\xi \in R$. In addition, by Lemma 2.5, we have

$$
\begin{aligned}
F_{2}(s, i)(\xi) \geqslant & \rho_{2} \int_{-\infty}^{\xi} e^{\Lambda_{2}^{-}(\xi-x)}\left[\alpha_{2} i_{-}(x)+f\left(s_{-}(x)\right) g\left(i_{-}(x-c \tau)\right)-(\mu+\gamma) i_{-}(x)\right] d x \\
& +\rho_{2} \int_{\xi}^{\infty} e^{\Lambda_{2}^{+}(\xi-x)}\left[\alpha_{2} i_{-}(x)+f\left(s_{-}(x)\right) g\left(i_{-}(x-c \tau)\right)-(\mu+\gamma) i_{-}(x)\right] d x \\
\geqslant & \rho_{2} \int_{-\infty}^{\xi} e^{\Lambda_{2}^{-}(\xi-x)}\left[-d_{2} i_{-}^{\prime \prime}(x)+c i_{-}^{\prime}(x)+\alpha_{2} i_{-}(x)\right] d x \\
& +\rho_{2} \int_{\xi}^{\infty} e^{\Lambda_{2}^{+}(\xi-x)}\left[-d_{2} i_{-}^{\prime \prime}(x)+c i_{-}^{\prime}(x)+\alpha_{2} i_{-}(x)\right] d x \\
\geqslant & i_{-}(\xi)+\rho_{2} d_{2} \min \left\{e^{\Lambda_{2}^{+}\left(\xi-\xi_{2}\right)}, e^{\Lambda_{2}^{-}\left(\xi-\xi_{2}\right)}\right\}\left[i_{-}^{\prime}\left(\xi_{2}+0\right)-i_{-}^{\prime}\left(\xi_{2}-0\right)\right] \\
\geqslant & i_{-}(\xi)
\end{aligned}
$$

for any $\xi \neq \xi_{2}$.

Since $g(i(\xi)) \leqslant g^{\prime}(0) i_{+}(\xi)$ and $f(s) \leqslant f(1)$, it follows from Lemma 2.3 that

$$
\begin{aligned}
F_{2}(s, i)(\xi) \leqslant & \rho_{2} \int_{-\infty}^{\xi} e^{\Lambda_{2}^{-}(\xi-x)}\left[\alpha_{2} i_{+}(x)+f\left(s_{+}(x)\right) g\left(i_{+}(x-c \tau)\right)-(\mu+\gamma) i_{+}(x)\right] d x \\
& +\rho_{2} \int_{\xi}^{\infty} e^{\Lambda_{2}^{+}(\xi-x)}\left[\alpha_{2} i_{+}(x)+f\left(s_{+}(x)\right) g\left(i_{+}(x-c \tau)\right)-(\mu+\gamma) i_{+}(x)\right] d x \\
\leqslant & \rho_{2} \int_{-\infty}^{\xi} e^{\Lambda_{2}^{-}(\xi-x)}\left[-d_{2} i_{+}^{\prime \prime}(x)+c i_{+}^{\prime}(x)+\alpha_{2} i_{+}(x)\right] d x \\
& +\rho_{2} \int_{\xi}^{\infty} e^{\Lambda_{2}^{+}(\xi-x)}\left[-d_{2} i_{+}^{\prime \prime}(x)+c i_{+}^{\prime}(x)+\alpha_{2} i_{+}(x)\right] d x \\
\leqslant & i_{+}(\xi)-\rho_{2} d_{2} \max \left\{e^{\Lambda_{2}^{-}\left(\xi-\xi_{0}\right)}, e^{\Lambda_{2}^{+}\left(\xi-\xi_{0}\right)}\right\} i_{+}^{\prime}\left(\xi_{0}-0\right) \\
\leqslant & i_{+}(\xi) .
\end{aligned}
$$

Hence, for any $(s, i) \in \Gamma, \xi \in R$,

$$
s_{-}(\xi) \leqslant F_{1}(s, i)(\xi) \leqslant s_{+}(\xi), \quad i_{-}(\xi) \leqslant F_{2}(s, i)(\xi) \leqslant i_{+}(\xi) .
$$

That is, $\mathrm{F}: \Gamma \rightarrow \Gamma$. 
Lemma 2.7. The operator $\mathrm{F}: \Gamma \rightarrow \Gamma$ is completely continuous.

Proof. First we prove the map $F$ is continuous. For any $\left(s_{1}, i_{1}\right) \in \Gamma,\left(s_{2}, i_{2}\right) \in \Gamma, \xi \in R$, by Lagrange's mean value theorem, we obtain

$$
\begin{aligned}
\mid f\left(s_{1}(\xi)\right) & g\left(i_{1}(\xi-c \tau)\right)-f\left(s_{2}(\xi)\right) g\left(i_{2}(\xi-c \tau)\right) \mid \\
\quad= & g\left(i_{1}(\xi-c \tau)\right)\left[f\left(s_{1}(\xi)\right)-f\left(s_{2}(\xi)\right)\right]+f\left(s_{2}(\xi)\right)\left[g\left(i_{1}(\xi-c \tau)\right)-g\left(i_{2}(\xi-c \tau)\right)\right] \mid \\
\quad & \leqslant f^{\prime}(\widetilde{s}) g\left(i_{1}(\xi-c \tau)\right)\left|s_{1}(\xi)-s_{2}(\xi)\right|+f(1) g^{\prime}(\widetilde{i})\left|i_{1}(\xi-c \tau)-i_{2}(\xi-c \tau)\right| \\
& \leqslant \sup _{0 \leqslant s \leqslant 1}\left\{f^{\prime}(s)\right\} g^{\prime}(0) B\left|s_{1}(\xi)-s_{2}(\xi)\right|+f(1) g^{\prime}(0)\left|i_{1}(\xi-c \tau)-i_{2}(\xi-c \tau)\right|,
\end{aligned}
$$

where $\widetilde{s}$ lies between $s_{1}(\xi)$ and $s_{2}(\xi)$, and $\widetilde{i}$ lies between $i_{1}(\xi-c \tau)$ and $i_{2}(\xi-c \tau)$. By the definition of $F_{1}$, for any $\xi \in R$,

$$
\begin{aligned}
& \left|F_{1}\left(s_{1}, i_{1}\right)(\xi)-F_{1}\left(s_{2}, i_{2}\right)(\xi)\right| e^{-\beta|\xi|} \\
& \leqslant \rho_{1} e^{-\beta|\xi|} \int_{-\infty}^{\xi}\left[\left(\alpha_{1}-\mu\right)\left|s_{1}(x)-s_{2}(x)\right|+\left|f\left(s_{1}\right) g\left(i_{1}\right)-f\left(s_{2}\right) g\left(i_{2}\right)\right|\right] e^{\Lambda_{1}^{-}(\xi-x)} d x \\
& +\rho_{1} e^{-\beta|\xi|} \int_{\xi}^{\infty}\left[\left(\alpha_{1}-\mu\right)\left|s_{1}(x)-s_{2}(x)\right|+\left|f\left(s_{1}\right) g\left(i_{1}\right)-f\left(s_{2}\right) g\left(i_{2}\right)\right|\right] e^{\Lambda_{1}^{+}(\xi-x)} d x \\
& \leqslant \rho_{1} l e^{-\beta|\xi|} \int_{-\infty}^{\xi}\left[\left|s_{1}(x)-s_{2}(x)\right|+\left|i_{1}(x-c \tau)-i_{2}(x-c \tau)\right|\right] e^{\wedge_{1}^{-}(\xi-x)} d x \\
& +\rho_{1} l e^{-\beta|\xi|} \int_{\xi}^{\infty}\left[\left|s_{1}(x)-s_{2}(x)\right|+\left|i_{1}(x-c \tau)-i_{2}(x-c \tau)\right|\right] e^{\Lambda_{1}^{+}(\xi-x)} d x \\
& \leqslant\left(1+e^{\beta c \tau}\right) \rho_{1} l e^{-\beta|\xi|}\left|\left(s_{1}-s_{2}, i_{1}-i_{2}\right)\right|_{\beta}\left[\int_{-\infty}^{\xi} e^{\Lambda_{1}^{-}(\xi-x)+\beta|x|} d x+\int_{\xi}^{\infty} e^{\Lambda_{1}^{+}(\xi-x)+\beta|x|} d x\right] \\
& =\left(1+e^{\beta c \tau}\right) \rho_{1} l\left|\left(s_{1}-s_{2}, i_{1}-i_{2}\right)\right|_{\beta} A(\xi),
\end{aligned}
$$

where

$$
\begin{gathered}
l:=\max \left\{\alpha_{1}-\mu+\sup _{0 \leqslant s \leqslant 1}\left\{f^{\prime}(s)\right\} g^{\prime}(0) B, f(1) g^{\prime}(0)\right\}, \\
A(\xi):=e^{-\beta|\xi|}\left[\int_{-\infty}^{\xi} e^{\Lambda_{1}^{-}(\xi-x)+\beta|x|} d x+\int_{\xi}^{\infty} e^{\Lambda_{1}^{+}(\xi-x)+\beta|x|} d x\right] .
\end{gathered}
$$

As $0<\beta<-\Lambda_{1}^{-}<\Lambda_{1}^{+}$, by L'Hospital's rule, we have

$$
A(-\infty)=\frac{1}{\beta+\Lambda_{1}^{+}}-\frac{1}{\beta+\Lambda_{1}^{-}}, \quad A(+\infty)=\frac{1}{\Lambda_{1}^{+}-\beta}+\frac{1}{\beta-\Lambda_{1}^{-}} .
$$

That is, $A(\xi)$ is bounded on R. Denote $\bar{A}=\sup _{\xi \in R}|A(\xi)|$. Hence,

$$
\sup _{\xi \in R}\left|F_{1}\left(s_{1}, i_{1}\right)(\xi)-F_{1}\left(s_{2}, i_{2}\right)(\xi)\right| e^{-\beta|\xi|} \leqslant\left(1+e^{\beta c \tau}\right) \rho_{1} l \bar{A}\left|\left(s_{1}, i_{1}\right)-\left(s_{2}, i_{2}\right)\right|_{\beta} .
$$

Similarly, we obtain

$$
\sup _{\xi \in R}\left|F_{2}\left(s_{1}, i_{1}\right)(\xi)-F_{2}\left(s_{2}, i_{2}\right)(\xi)\right| e^{-\beta|\xi|} \leqslant Q\left|\left(s_{1}, i_{1}\right)-\left(s_{2}, i_{2}\right)\right|_{\beta},
$$

where $\mathrm{Q}$ is a constant. Hence, $\mathrm{F}$ is continuous on $\Gamma$. 
Next, we prove that $F$ is compact. For any $(s, i) \in \Gamma$, we get

$$
\begin{aligned}
\left|\frac{\mathrm{d}}{\mathrm{d} \xi} \mathrm{F}_{1}(s, i)(\xi)\right|= & \mid \rho_{1} \Lambda_{1}^{-} \int_{-\infty}^{\xi} e^{\Lambda_{1}^{-}(\xi-x)}\left[\alpha_{1} s(x)-f(s(x)) g(i(x-c \tau))-\mu s(x)+\mu\right] \mathrm{d} x \\
& +\rho_{1} \Lambda_{1}^{+} \int_{\xi}^{\infty} e^{\Lambda_{1}^{+}(\xi-x)}\left[\alpha_{1} s(x)-f(s(x)) g(i(x-c \tau))-\mu s(x)+\mu\right] \mathrm{d} x \mid \\
\leqslant & -\rho_{1} \Lambda_{1}^{-} \int_{-\infty}^{\xi} e^{\Lambda_{1}^{-}(\xi-x)}\left[\left(\alpha_{1}-\mu\right) s(x)+\mu+f(s(x)) g(i(x-c \tau))\right] \mathrm{d} x \\
& +\rho_{1} \Lambda_{1}^{+} \int_{\xi}^{\infty} e^{\Lambda_{1}^{+}(\xi-x)}\left[\left(\alpha_{1}-\mu\right) s(x)+\mu+f(s(x)) g(i(x-c \tau))\right] \mathrm{d} x \\
\leqslant & -\rho_{1}\left(\alpha_{1}+f(1) g^{\prime}(0) B\right)\left(\Lambda_{1}^{-} \int_{-\infty}^{\xi} e^{\Lambda_{1}^{-}(\xi-x)} \mathrm{d} x-\Lambda_{1}^{+} \int_{\xi}^{\infty} e^{\Lambda_{1}^{+}(\xi-x)} \mathrm{d} x\right) \\
= & 2 \rho_{1}\left[\alpha_{1}+f(1) g^{\prime}(0) B\right] .
\end{aligned}
$$

Since $f(s(x)) g(i(x-c \tau)) \leqslant f(1) g^{\prime}(0) B$ and $\left(\alpha_{2}-\mu-\gamma\right) i$ is increasing with respect to $i$, we obtain

$$
\begin{aligned}
\left|\frac{\mathrm{d}}{\mathrm{d} \xi} \mathrm{F}_{2}(\mathrm{~s}, \mathrm{i})(\xi)\right|= & \mid \rho_{2} \Lambda_{2}^{-} \int_{-\infty}^{\xi} e^{\Lambda_{2}^{-}(\xi-x)}\left[f(s(x)) g(i(x-c \tau))+\left(\alpha_{2}-\mu-\gamma\right) i(x)\right] \mathrm{d} x \\
& +\rho_{2} \Lambda_{2}^{+} \int_{\xi}^{\infty} e^{\Lambda_{2}^{+}(\xi-x)}\left[f(s(x)) g(i(x-c \tau))+\left(\alpha_{2}-\mu-\gamma\right) i(x)\right] \mathrm{d} x \mid \\
\leqslant & -\rho_{2} B\left(\alpha_{2}+f(1) g^{\prime}(0)\right)\left(\Lambda_{2}^{-} \int_{-\infty}^{\xi} e^{\Lambda_{2}^{-}(\xi-x)} \mathrm{d} x-\Lambda_{2}^{+} \int_{\xi}^{\infty} e^{\Lambda_{2}^{+}(\xi-x)} \mathrm{d} x\right) \\
= & 2 \rho_{2} B\left[\alpha_{2}+f(1) g^{\prime}(0)\right] .
\end{aligned}
$$

In addition, $F(\Gamma)$ is uniformly bounded on R. Hence, for each $n \in N$, by using Ascoli-Arzela theorem, $F(\Gamma)$ is precompact on $[-n, n]$. Using the standard diagonal method, $F(\Gamma)$ is precompact on R. Hence, $F: \Gamma \rightarrow \Gamma$ is completely continuous.

\section{Proof of main results}

In this section, we first establish the existence of a nonnegative solution of system (1.5) satisfying (1.4). Proof of Theorem 1.1. As $c>c^{*}$, in view of Lemmas 2.6 and 2.7, it follows from the Schauder's fixed point theorem that $F$ has a fixed point $(s, i) \in \Gamma$. Hence there exists a nonnegative solution $(s(\xi), i(\xi))$ of system (1.5) satisfying

$$
s_{-}(\xi) \leqslant s(\xi) \leqslant s_{+}(\xi)=1, \quad i_{-}(\xi) \leqslant i(\xi) \leqslant i_{+}(\xi) \leqslant B, \quad \xi \in R .
$$

Together with the definitions of $s_{+}, s_{-}, i_{+}$and $i_{-}$, it follows that

$$
(s, i)(-\infty)=(1,0), \quad \lim _{\xi \rightarrow-\infty} i(\xi) e^{-\lambda_{1} \xi}=1 .
$$

Applying the L'Hospital theorem to the maps $F_{1}$ and $F_{2}$, it is easy to show that

$$
\lim _{\xi \rightarrow-\infty}\left(s^{\prime}(\xi), i^{\prime}(\xi)\right)=(0,0) .
$$

Furthermore, we claim that $0<s(\xi)<1$ and $0<i(\xi)<B$ for all $\xi \in R$. First, we prove $s(\xi)>0$ on R. For contradiction, we assume that $s\left(\widetilde{\xi_{1}}\right)=0$ for some $\widetilde{\xi_{1}} \in R$. Then $s^{\prime}\left(\widetilde{\xi_{1}}\right)=0$ and $s^{\prime \prime}\left(\widetilde{\xi_{1}}\right) \geqslant 0$. However, it follows from the first equation of (1.5) that $s^{\prime \prime}\left(\widetilde{\xi_{1}}\right)<0$. Next, we certify $i(\xi)>0$ on R. If not, 
denote $\widetilde{\xi_{2}}=\inf \{\xi \in R \mid i(\xi)=0\}$, then $i\left(\widetilde{\xi_{2}}\right)=0, i^{\prime}\left(\widetilde{\xi_{2}}\right)=0$ and $i^{\prime \prime}\left(\widetilde{\xi_{2}}\right) \geqslant 0$. But, it follows from the second equation of (1.5) that $i^{\prime \prime}\left(\widetilde{\xi}_{2}\right)<0$. Similarly, we have $s(\xi)<1$ on R. Finally, we show $i(\xi)<B$ on R. If not, we assume that $i\left(\widetilde{\xi_{3}}\right)=B$ for some $\widetilde{\xi_{3}} \in R$. Then $i^{\prime}\left(\widetilde{\xi_{3}}\right)=0$ and $i^{\prime \prime}\left(\widetilde{\xi_{3}}\right) \leqslant 0$. However, it follows from the second equation of $(1.5)$ and $\left(\mathrm{A}_{3}\right)$ that $i^{\prime \prime}\left(\widetilde{\xi}_{3}\right)>0$.

Now we verify that $(s, i)(+\infty)=\left(s^{*}, i^{*}\right)$. For this purpose, we rewrite (1.5) as a system

$$
\left\{\begin{array}{l}
s^{\prime}(\xi)=w(\xi) \\
d_{1} w^{\prime}(\xi)=c w(\xi)+\mu(s(\xi)-1)+f(s(\xi)) g(i(\xi-c \tau)) \\
i^{\prime}(\xi)=y(\xi) \\
d_{2} y^{\prime}(\xi)=c y(\xi)-f(s(\xi)) g(i(\xi-c \tau))+(\mu+\gamma) i(\xi) .
\end{array}\right.
$$

Construct the Lyapunov functional $\mathrm{U}$ by

$$
U(s, w, i, y)=U_{1}(s, w, i, y)+U_{2}(s, w, i, y)+c(\mu+\gamma) i^{*} U_{3}(s, w, i, y),
$$

where

$$
\begin{aligned}
& u_{1}(s, w, i, y)=-\left(d_{1} w-c s-f\left(s^{*}\right) \frac{d_{1} w}{f(s)}+c f\left(s^{*}\right) \int_{s^{*}}^{s} \frac{1}{f(\sigma)} d \sigma\right), \\
& u_{2}(s, w, i, y)=-\left(d_{2} y-c i-g\left(i^{*}\right) \frac{d_{2} y}{g(i)}+c g\left(i^{*}\right) \int_{i^{*}}^{i} \frac{1}{g(\sigma)} d \sigma\right), \\
& u_{3}(s, w, i, y)=\int_{0}^{\tau}\left[\frac{g(i(\xi-c \theta))}{g\left(i^{*}\right)}-1-\ln \frac{g(i(\xi-c \theta))}{g\left(i^{*}\right)}\right] d \theta .
\end{aligned}
$$

Let $\chi(\xi):=(s(\xi), w(\xi), i(\xi), y(\xi))$ be the solution of (3.1). Then

$$
\begin{aligned}
& \frac{d}{d \xi} U_{1}(\chi(\xi))=-d_{1} w^{\prime}(\xi)+c s^{\prime}(\xi)+d_{1} f\left(s^{*}\right)\left[\frac{w^{\prime}(\xi)}{f(s(\xi))}-\frac{w(\xi) f^{\prime}(s(\xi)) s^{\prime}(\xi)}{f^{2}(s(\xi))}\right]-\frac{c f\left(s^{*}\right) s^{\prime}(\xi)}{f(s(\xi))} \\
& =-d_{1} f\left(s^{*}\right) f^{\prime}(s(\xi)) \frac{w^{2}(\xi)}{f^{2}(s(\xi))}+[\mu(1-s(\xi))-f(s(\xi)) g(i(\xi-c \tau))]\left(1-\frac{f\left(s^{*}\right)}{f(s(\xi))}\right) \\
& =-d_{1} f\left(s^{*}\right) f^{\prime}(s(\xi)) \frac{w^{2}(\xi)}{f^{2}(s(\xi))}+\left[\mu\left(s^{*}-s(\xi)\right)+\mu\left(1-s^{*}\right)\right. \\
& -f(s(\xi)) g(i(\xi-c \tau))]\left(1-\frac{f\left(s^{*}\right)}{f(s(\xi))}\right) \\
& \frac{d}{d \xi} u_{2}(x(\xi))=-d_{2} y^{\prime}(\xi)+c i^{\prime}(\xi)+d_{2} g\left(i^{*}\right) \frac{y^{\prime}(\xi) g(i(\xi))-y(\xi) g^{\prime}(i(\xi)) i^{\prime}(\xi)}{g^{2}(i(\xi))}-\frac{c g\left(i^{*}\right) i^{\prime}(\xi)}{g(i(\xi))} \\
& =f(s(\xi)) g(i(\xi-c \tau))+g\left(i^{*}\right) g(\mathfrak{i}(\xi)) \frac{c y(\xi)-f(s(\xi)) g(i(\xi-c \tau))+(\gamma+\mu) \mathfrak{i}(\xi)}{g^{2}(\mathfrak{i}(\xi))} \\
& -(\gamma+\mu) i(\xi)-d_{2} g\left(i^{*}\right) g^{\prime}(i(\xi)) \frac{y^{2}(\xi)}{g^{2}(i(\xi))}-c g\left(i^{*}\right) \frac{y(\xi)}{g(i(\xi))} \\
& =-d_{2} g\left(i^{*}\right) g^{\prime}(\mathfrak{i}(\xi)) \frac{y^{2}(\xi)}{g^{2}(\mathfrak{i}(\xi))}+[f(s(\xi)) g(i(\xi-c \tau))-(\gamma+\mu) i(\xi)]\left(1-\frac{g\left(i^{*}\right)}{g(i(\xi))}\right),
\end{aligned}
$$

and

$$
\begin{aligned}
\frac{\mathrm{d}}{\mathrm{d} \xi} \mathrm{U}_{3}(\chi(\xi)) & =\int_{0}^{\tau} \frac{\mathrm{d}}{\mathrm{d} \xi}\left[\frac{g(i(\xi-c \theta))}{g\left(i^{*}\right)}-1-\ln \frac{g(i(\xi-c \theta))}{g\left(i^{*}\right)}\right] d \theta \\
& =-\frac{1}{\mathrm{c}} \int_{0}^{\tau} \frac{\mathrm{d}}{\mathrm{d} \theta}\left[\frac{g(i(\xi-c \theta))}{g\left(i^{*}\right)}-1-\ln \frac{g(i(\xi-c \theta))}{g\left(i^{*}\right)}\right] d \theta
\end{aligned}
$$




$$
\begin{aligned}
& =-\frac{1}{c}\left[\frac{g(i(\xi-c \tau))}{g\left(i^{*}\right)}-\frac{g(i(\xi))}{g\left(i^{*}\right)}-\ln \frac{g(i(\xi-c \tau))}{g(i(\xi))}\right] \\
& =-\frac{1}{c}\left[\frac{g(i(\xi-c \tau))}{g\left(i^{*}\right)}-\frac{g(i(\xi))}{g\left(i^{*}\right)}-\ln \frac{f\left(s^{*}\right)}{f(s(\xi))}-\ln \frac{f(s(\xi)) g(i(\xi-c \tau))}{f\left(s^{*}\right) g(i(\xi))}\right] .
\end{aligned}
$$

Consequently,

$$
\begin{aligned}
\frac{d}{d \xi} U(\chi(\xi))= & -d_{1} f\left(s^{*}\right) f^{\prime}(s(\xi)) \frac{w^{2}(\xi)}{f^{2}(s(\xi))}-d_{2} g\left(i^{*}\right) g^{\prime}(i(\xi)) \frac{y^{2}(\xi)}{g^{2}(i(\xi))} \\
& +\mu\left(s^{*}-s(\xi)\right)\left(1-\frac{f\left(s^{*}\right)}{f(s(\xi))}\right)+\mu\left(1-s^{*}\right)\left(1-\frac{f\left(s^{*}\right)}{f(s(\xi))}\right) \\
& +f\left(s^{*}\right) g(i(\xi-c \tau))-g\left(i^{*}\right) \frac{f(s(\xi)) g(i(\xi-c \tau))}{g(i(\xi))}-(\gamma+\mu) i(\xi)\left(1-\frac{g\left(i^{*}\right)}{g(i(\xi))}\right) \\
& +(\gamma+\mu) i^{*}\left[-\frac{g(i(\xi-c \tau))}{g\left(i^{*}\right)}+\frac{g(i(\xi))}{g\left(i^{*}\right)}+\ln \frac{f\left(s^{*}\right)}{f(s(\xi))}+\ln \frac{f(s(\xi)) g(i(\xi-c \tau))}{f\left(s^{*}\right) g(i(\xi))}\right] .
\end{aligned}
$$

Note that $\mu\left(1-s^{*}\right)=(\gamma+\mu) i^{*}$ and $f\left(s^{*}\right) g\left(i^{*}\right)=(\gamma+\mu) i^{*}$. Thus, for any $\xi \in R$,

$$
\begin{aligned}
\frac{d}{d \xi} U(\chi(\xi))= & -d_{1} f\left(s^{*}\right) f^{\prime}(s(\xi)) \frac{w^{2}(\xi)}{f^{2}(s(\xi))}-d_{2} g\left(i^{*}\right) g^{\prime}(i(\xi)) \frac{y^{2}(\xi)}{g^{2}(i(\xi))} \\
& +\mu\left(s^{*}-s(\xi)\right)\left(1-\frac{f\left(s^{*}\right)}{f(s(\xi))}\right)+(\gamma+\mu) i^{*}\left(1-\frac{f\left(s^{*}\right)}{f(s(\xi))}+\ln \frac{f\left(s^{*}\right)}{f(s(\xi))}\right) \\
& +(\gamma+\mu) i^{*} \frac{g(i(\xi))}{g\left(i^{*}\right)}-(\gamma+\mu) i^{*}-(\gamma+\mu) i(\xi)\left(1-\frac{g\left(i^{*}\right)}{g(i(\xi))}\right) \\
& +(\gamma+\mu) i^{*}\left[1-\frac{f(s(\xi)) g(i(\xi-c \tau))}{f\left(s^{*}\right) g(i(\xi))}+\ln \frac{f(s(\xi)) g(i(\xi-c \tau))}{f\left(s^{*}\right) g(i(\xi))}\right] \\
= & -d_{1} f\left(s^{*}\right) f^{\prime}(s(\xi)) \frac{w^{2}(\xi)}{f^{2}(s(\xi))}-d_{2} g\left(i^{*}\right) g^{\prime}(i(\xi)) \frac{y^{2}(\xi)}{g^{2}(i(\xi))} \\
& +\mu\left(s^{*}-s(\xi)\right)\left(1-\frac{f\left(s^{*}\right)}{f(s(\xi))}\right)+(\gamma+\mu) i^{*}\left(1-\frac{f\left(s^{*}\right)}{f(s(\xi))}+\ln \frac{f\left(s^{*}\right)}{f(s(\xi))}\right) \\
& -(\gamma+\mu) i^{*}\left(1-\frac{g\left(i^{*}\right)}{g(i(\xi))}\right)\left(\frac{i(\xi)}{i^{*}}-\frac{g(i(\xi))}{g\left(i^{*}\right)}\right) \\
& +(\gamma+\mu) i^{*}\left[1-\frac{f(s(\xi)) g(i(\xi-c \tau))}{f\left(s^{*}\right) g(i(\xi))}+\ln \frac{f(s(\xi)) g(i(\xi-c \tau))}{f\left(s^{*}\right) g(i(\xi))}\right] .
\end{aligned}
$$

Since $f^{\prime}(s)>0$ for any $s \in[0,+\infty)$,

$$
\left(s^{*}-s(\xi)\right)\left(1-\frac{f\left(s^{*}\right)}{f(s(\xi))}\right) \leqslant 0, \quad \xi \in R .
$$

Note that $1-\xi+\ln \xi \leqslant 0$ for any $\xi>0$. Hence,

$$
\begin{array}{r}
1-\frac{f\left(s^{*}\right)}{f(s(\xi))}+\ln \frac{f\left(s^{*}\right)}{f(s(\xi))} \leqslant 0, \quad \xi \in R, \\
1-\frac{f(s(\xi)) g(i(\xi-c \tau))}{f\left(s^{*}\right) g(i(\xi))}+\ln \frac{f(s(\xi)) g(i(\xi-c \tau))}{f\left(s^{*}\right) g(i(\xi))} \leqslant 0, \quad \xi \in R .
\end{array}
$$

Furthermore, since the function $g(i)$ is concave,

$$
\left\{\begin{array}{l}
\frac{g(i)}{g\left(i^{*}\right)} \geqslant \frac{i}{i^{*}}, \quad \text { if } 0<i \leqslant i^{*} \\
\frac{g(i)}{g\left(i^{*}\right)} \leqslant \frac{i}{i^{*}}, \quad \text { if } i \geqslant i^{*}
\end{array}\right.
$$


which implies that

$$
\left(1-\frac{g\left(i^{*}\right)}{g(i(\xi))}\right)\left(\frac{i(\xi)}{i^{*}}-\frac{g(i(\xi))}{g\left(i^{*}\right)}\right) \geqslant 0, \quad \xi \in R
$$

Hence,

$$
\frac{\mathrm{d}}{\mathrm{d} \xi} \mathrm{U}(\chi(\xi)) \leqslant 0, \quad \xi \in \mathrm{R} .
$$

Let

$$
\begin{aligned}
& \left\{s_{n}(\xi)=s(\xi+n)\right\}_{n} \geqslant 0 \text { and }\left\{i_{n}(\xi)=i(\xi+n)\right\}_{n} \geqslant 0, \\
& \left\{w_{\mathfrak{n}}(\xi)=w(\xi+n)\right\}_{\mathfrak{n} \geqslant 0} \text { and }\left\{y_{\mathfrak{n}}(\xi)=y(\xi+n)\right\}_{\mathfrak{n} \geqslant 0} \text {. }
\end{aligned}
$$

Since $s_{n}, i_{n}, s_{n}^{\prime}, i_{n}^{\prime} s_{n}^{\prime \prime}$, and $i_{n}^{\prime \prime}$ are uniformly bounded in $R$, up to a subsequence, we can assume that $s_{n}, w_{n}, i_{n}$, and $y_{n}$ converge to some nonnegative functions $s_{\infty}, w_{\infty}, i_{\infty}$, and $y_{\infty}$, which satisfy (3.1). Besides, since $U(\chi(\xi))$ is non-increasing on $\xi$ and bounded below, there exists some $v \in R$ such that $\lim _{\xi \rightarrow+\infty} \mathrm{U}(\chi(\xi))=v$. Further,

$$
\lim _{n \rightarrow+\infty} \mathrm{u}\left(s_{n}, w_{n}, i_{n}, y_{n}\right)(\xi)=\lim _{n \rightarrow+\infty} u(s, w, i, y)\left(\xi+\xi_{n}\right)=v
$$

for any $\xi \in \mathrm{R}$. By (3.2) and Lebegue dominated convergence theorem, we obtain

$$
\lim _{n \rightarrow+\infty} \mathrm{u}\left(s_{n}, w_{n}, i_{n}, y_{n}\right)(\xi)=u\left(s_{\infty}, w_{\infty}, i_{\infty}, y_{\infty}\right)(\xi), \quad \xi \in R
$$

Thus

$$
\mathrm{U}\left(\mathrm{s}_{\infty}, w_{\infty}, i_{\infty}, \mathrm{y}_{\infty}\right)(\xi)=v, \quad \xi \in \mathrm{R}
$$

Therefore,

$$
\frac{\mathrm{d}}{\mathrm{d} \xi} \mathrm{u}\left(s_{\infty}, w_{\infty}, i_{\infty}, \mathrm{y}_{\infty}\right)=0
$$

which, together with (3.3), yields

$$
\mathrm{s}_{\infty}(\xi) \equiv \mathrm{s}^{*}, \quad w_{\infty}(\xi) \equiv 0, \quad i_{\infty}(\xi) \equiv i^{*}, \quad y_{\infty}(\xi) \equiv 0, \quad \xi \in R .
$$

So, for any $\xi \in[0,1], s_{n}(\xi)$ converges uniformly to $s^{*}$ as $n \rightarrow \infty$. That is, for any $\xi \in[0,1]$ and $\in \in(0,1)$, there exists a positive number $\mathrm{N}$ such that

$$
\left|s_{n}(\xi)-s^{*}\right|<\epsilon, \quad n \geqslant N,
$$

then $\left|s(\xi+n)-s^{*}\right|<\epsilon$ and

$$
\left|s(\xi)-s^{*}\right|<\epsilon, \quad \xi \geqslant N .
$$

Hence, $\lim _{\xi \rightarrow+\infty} s(\xi)=s^{*}$. Similarly, $\lim _{\xi \rightarrow+\infty} i(\xi)=i^{*}, \lim _{\xi \rightarrow+\infty} s^{\prime}(\xi)=0$, and $\lim _{\xi \rightarrow+\infty} i^{\prime}(\xi)=0$. This completes the proof.

In the following, we prove the non-existence of nonnegative solution of system (1.5). Next, we first give the following result.

Lemma 3.1. Assume that $\mathrm{R}_{0}>1$ and $\mathrm{c} \in\left(0, \mathrm{c}^{*}\right)$ or $\mathrm{R}_{0}=1$. If system (1.5) exists a nontrivial and nonnegative solution $(s(\xi), i(\xi))$ satisfying $(1.4)$, then $0<s(\xi)<1$ and $i(\xi)>0$ for any $\xi \in R$, and $\lim _{\xi \rightarrow-\infty} i^{\prime}(\xi)=$ $\lim _{\xi \rightarrow+\infty} i^{\prime}(\xi)=0$.

Proof. The proofs of $s(\xi)>0$ and $i(\xi)>0$ are similar to that of Theorem 1.1, we omit their proof. Next, we prove $s(\xi)<1$ for any $\xi \in R$. On the contrary, note that $s(+\infty)=s^{*}$ and $0<s^{*}<1$, denote $\widetilde{\xi_{0}}=\sup \{\xi \in R \mid s(\xi)=1\}$, then $s\left(\widetilde{\xi_{0}}\right)=1$ and $s^{\prime}\left(\widetilde{\xi_{0}}\right) \leqslant 0$. If $s^{\prime}\left(\widetilde{\xi_{0}}\right)=0$, then $s^{\prime \prime}\left(\widetilde{\xi_{0}}\right) \leqslant 0$. However, it follows 
from the first equation of (1.5) that $s^{\prime \prime}\left(\widetilde{\varepsilon_{0}}\right)>0$. If $s^{\prime}\left(\widetilde{\xi_{0}}\right)<0$, note that $s(-\infty)=1$, then there exists an $\widehat{\xi}<\widetilde{\xi_{0}}$ such that $s(\widehat{\xi})>1, s^{\prime}(\widehat{\xi})=0$ and $s^{\prime \prime}(\widehat{\xi}) \leqslant 0$. But, it follows from the first equation of (1.5) that $s^{\prime \prime}(\widehat{\xi})>0$. It is a contradiction.

Now, we claim that $\lim _{\xi \rightarrow+\infty} i^{\prime}(\xi)=0$. Let $\limsup _{\xi \rightarrow+\infty} i^{\prime}(\xi)=a, \liminf _{\xi \rightarrow+\infty} i^{\prime}(\xi)=b$, then $a \geqslant b$. If $a>b$, there exist $\xi_{n}, \eta_{n} \rightarrow \infty$ such that

$$
\begin{array}{ll}
\lim _{n \rightarrow \infty} i^{\prime}\left(\xi_{n}\right)=a, & \lim _{n \rightarrow \infty} i^{\prime \prime}\left(\xi_{n}\right)=0, \\
\lim _{n \rightarrow \infty} i^{\prime}\left(\eta_{n}\right)=b, & \lim _{n \rightarrow \infty} i^{\prime \prime}\left(\eta_{n}\right)=0 .
\end{array}
$$

It follows from the second equation of (1.5) that

$$
c a=f\left(s^{*}\right) g\left(i^{*}\right)-(\mu+\gamma) i^{*}, \quad c b=f\left(s^{*}\right) g\left(i^{*}\right)-(\mu+\gamma) i^{*} .
$$

Note that $c>0$, we have $a=b$, a contradiction. Hence $\lim _{\xi \rightarrow+\infty} i^{\prime}(\xi)$ exists, and $\lim _{\xi \rightarrow+\infty} i^{\prime}(\xi)=\lim _{\xi \rightarrow+\infty} \frac{i(\xi)}{\xi}=$ 0 . Similarly, $\lim _{\xi \rightarrow-\infty} i^{\prime}(\xi)=0$. This completes the proof.

Proof of Theorem 1.2. For contradiction, assume that there exists a nonnegative solution $(s(\xi), i(\xi))$ of system (1.5) satisfying (1.4).

We firstly consider $R_{0}=1$. It follows from the second equation of (1.5) that

$$
c \int_{-\infty}^{+\infty} i^{\prime}(\xi) d \xi=d_{2} \int_{-\infty}^{+\infty} i^{\prime \prime}(\xi) d \xi+\int_{-\infty}^{+\infty} f(s(\xi)) g(i(\xi-c \tau)) d \xi-(\mu+\gamma) \int_{-\infty}^{+\infty} i(\xi) d \xi .
$$

Note that $i(-\infty)=0, i(+\infty)=i^{*}, i^{\prime}( \pm \infty)=0$. It follows from $R_{0}=1$ that

$$
(\mu+\gamma) \int_{-\infty}^{+\infty} i(\xi) d \xi=-c i^{*}+\int_{-\infty}^{+\infty} f(s(\xi)) g(i(\xi-c \tau)) d \xi<f(1) g^{\prime}(0) \int_{-\infty}^{+\infty} i(\xi-c \tau) d \xi=(\mu+\gamma) \int_{-\infty}^{+\infty} i(\xi) d \xi .
$$

This is a contradiction.

Secondly, we discuss the case that $R_{0}>1$ and $c \in\left(0, c^{*}\right)$. For any $\epsilon \in\left(0, g^{\prime}(0)\right)$, there exists a small positive number $\delta_{0}$ such that

$$
\frac{g(i)}{i} \geqslant g^{\prime}(0)-\epsilon, \quad 0<i<\delta_{0} .
$$

Note that $f(s(\xi)) \rightarrow f(1)$ and $i(\xi) \rightarrow 0$ as $\xi \rightarrow-\infty$. It follows from $R_{0}>1$ that there exists $\widetilde{\xi}<0$ such that $i(\xi)<\delta_{0}$ and

$$
f(s(\xi))>f(1)-\frac{f(1) g^{\prime}(0)-(\mu+\gamma)}{2 g^{\prime}(0)}=\frac{f(1) g^{\prime}(0)+\gamma+\mu}{2 g^{\prime}(0)}
$$

for any $\xi<\widetilde{\xi}$. Then, for any $\xi<\widetilde{\xi}$,

$$
\begin{aligned}
c i^{\prime}(\xi) & =d_{2} i^{\prime \prime}(\xi)+f(s(\xi)) g(i(\xi-c \tau))-(\mu+\gamma) i(\xi) \\
& \geqslant d_{2} i^{\prime \prime}(\xi)+\frac{f(1) g^{\prime}(0)+\gamma+\mu}{2 g^{\prime}(0)}\left(g^{\prime}(0)-\epsilon\right) i(\xi-c \tau)-(\mu+\gamma) i(\xi),
\end{aligned}
$$

which implies

$$
c i^{\prime}(\xi) \geqslant d_{2} i^{\prime \prime}(\xi)+\frac{f(1) g^{\prime}(0)+\gamma+\mu}{2}[i(\xi-c \tau)-i(\xi)]+\frac{f(1) g^{\prime}(0)-\gamma-\mu}{2} i(\xi) .
$$

Denote $k(\xi):=\int_{-\infty}^{\xi} i(x) d x$. Integrating the two sides of (3.5) from $-\infty$ to $\xi$ with $\xi<\widetilde{\xi}$, we have

$$
\frac{f(1) g^{\prime}(0)-\gamma-\mu}{2} k(\xi) \leqslant c i(\xi)-d_{2} i^{\prime}(\xi)+\frac{f(1) g^{\prime}(0)+\gamma+\mu}{2} \int_{\xi-c \tau}^{\xi} i(x) d x .
$$


Note that

$$
\begin{aligned}
\int_{-\infty}^{\xi} \int_{x-c \tau}^{x} i(s) \mathrm{d} s \mathrm{~d} x & =\int_{-\infty}^{\xi-c \tau} \int_{s}^{s+c \tau} i(s) \mathrm{d} x \mathrm{~d} s+\int_{\xi-c \tau}^{\xi} \int_{s}^{\xi} i(s) \mathrm{d} x \mathrm{~d} s \\
& =c \tau \int_{-\infty}^{\xi-c \tau} i(s) \mathrm{d} s+\int_{\xi-c \tau}^{\xi} i(s)(\xi-s) \mathrm{d} s \leqslant c \tau \int_{-\infty}^{\xi} i(s) \mathrm{d} s=c \tau k(\xi) .
\end{aligned}
$$

Integrating the both sides of (3.6) from $-\infty$ to $\xi$ yields

$$
\begin{aligned}
\frac{f(1) g^{\prime}(0)-\gamma-\mu}{2} \int_{-\infty}^{\xi} k(s) d s+d_{2} i(\xi) & \leqslant c \int_{-\infty}^{\xi} i(s) d s+\frac{f(1) g^{\prime}(0)+\gamma+\mu}{2} \int_{-\infty}^{\xi} \int_{x-c \tau}^{x} i(s) d s d x \\
& \leqslant c k(\xi)+\frac{f(1) g^{\prime}(0)+\gamma+\mu}{2} \operatorname{cck}(\xi)=m k(\xi),
\end{aligned}
$$

where $m=c+\frac{f(1) g^{\prime}(0)+\gamma+\mu}{2} c \tau$. Since $k(\xi)$ is increasing in $\xi$, for any $\eta>0$,

$$
\eta k(\xi-\eta)<\int_{\xi-\eta}^{\xi} k(s) d s<\int_{-\infty}^{\xi} k(s) d s,
$$

which, together with (3.7), for a large $\eta_{0}>0$, yields

$$
k\left(\xi-\eta_{0}\right)<\frac{k(\xi)}{2}, \quad \xi<\widetilde{\xi} .
$$

Define $\mu_{0}=\frac{\ln 2}{\eta_{0}}>0$ and $p(x)=k(x) e^{-\mu_{0} x}$. Then

$$
\mathrm{p}\left(\xi-\eta_{0}\right)=\mathrm{k}\left(\xi-\eta_{0}\right) e^{-\mu_{0}\left(\xi-\eta_{0}\right)}<\frac{k(\xi)}{2} e^{-\mu_{0} \xi} e^{\mu_{0} \eta_{0}}=p(\xi), \quad \xi<\widetilde{\xi},
$$

which implies that $p(x)$ is bounded on $(-\infty, \tilde{\xi}]$. It follows from $\lim _{x \rightarrow+\infty} p(x)=0$ that there exists $p_{0}>0$ satisfying $p(x) \leqslant p_{0}$ for any $x \in R$. This implies

$$
k(x) \leqslant p_{0} e^{\mu_{0} x}, \quad x \in R .
$$

It follows from (3.7) that

$$
\mathrm{d}_{2} i(\xi) \leqslant m k(\xi), \quad \xi<\widetilde{\xi} .
$$

This, together with (3.8), implies

$$
i(\xi) e^{-\mu_{0} \xi} \leqslant \frac{m}{d_{2}} p_{0}, \quad \xi<\widetilde{\xi} .
$$

By (3.6) and (3.8), for any $\xi<\widetilde{\xi}$, we obtain

$$
\begin{aligned}
d_{2} i^{\prime}(\xi) & \leqslant c i(\xi)+\frac{f(1) g^{\prime}(0)+\gamma+\mu}{2} \int_{\xi-c \tau}^{\xi} i(x) d x \\
& =c i(\xi)+\frac{f(1) g^{\prime}(0)+\gamma+\mu}{2}(k(\xi)-k(\xi-c \tau)) \\
& \leqslant c i(\xi)+\left(f(1) g^{\prime}(0)+\gamma+\mu\right) p_{0} e^{\mu_{0} \xi} .
\end{aligned}
$$

Thus, $\left|i^{\prime}(\xi)\right| e^{-\mu_{0} \xi}$ is bounded on $(-\infty, \widetilde{\xi}]$. Further, it follows from (3.5) that $\left|i^{\prime \prime}(\xi)\right| e^{-\mu_{0} \xi}$ is bounded on $(-\infty, \tilde{\xi}]$. Note that $\lim _{\xi \rightarrow+\infty} i(\xi), \lim _{\xi \rightarrow+\infty} i^{\prime}(\xi), \lim _{\xi \rightarrow+\infty} i^{\prime \prime}(\xi)$ all exist. Hence,

$$
\sup _{\xi \in R}\left\{\mathfrak{i}(\xi) e^{-\mu_{0} \xi}\right\}<\infty, \quad \sup _{\xi \in R}\left\{i^{\prime}(\xi) e^{-\mu_{0} \xi}\right\}<\infty, \quad \sup _{\xi \in R}\left\{i^{\prime \prime}(\xi) e^{-\mu_{0} \xi}\right\}<\infty .
$$


For $\lambda \in \mathbb{C}$ with $0<\operatorname{Re} \lambda<\mu_{0}$, we define a two-sided Laplace transform of $i$ by

$$
\mathrm{L}(\lambda)=\int_{-\infty}^{+\infty} e^{-\lambda \xi} \mathfrak{i}(\xi) \mathrm{d} \xi .
$$

It follows from the second equation of (1.5) that

$$
d_{2} i^{\prime \prime}(\xi)-c i^{\prime}(\xi)+f(1) g^{\prime}(0) i(\xi-c \tau)-(\mu+\gamma) \mathfrak{i}(\xi)=F_{1}(\xi),
$$

where

$$
F_{1}(\xi):=f(1) g^{\prime}(0) i(\xi-c \tau)-f(s(\xi)) g(i(\xi-c \tau))
$$

Further,

$$
\Delta(\lambda, c) \mathrm{L}(\lambda)=\int_{-\infty}^{+\infty} e^{-\lambda \xi} \mathrm{F}_{1}(\xi) \mathrm{d} \xi .
$$

When $0<i<\delta_{0}$, it follows from (3.4) that

$$
\begin{aligned}
F_{1}(\xi) & =\left(f(1) g^{\prime}(0)-f(s(\xi)) \frac{g(i(\xi-c \tau))}{i(\xi-c \tau)}\right) i(\xi-c \tau) \\
& \leqslant\left(\frac{f(1) g^{\prime}(0)-f(s(\xi)) \frac{g(i(\xi-c \tau))}{i(\xi-c \tau)}+i(\xi-c \tau)}{2}\right)^{2} \\
& \leqslant\left(f(1) g^{\prime}(0)-f(s(\xi))\left(g^{\prime}(0)-\epsilon\right)+i(\xi-c \tau)\right)^{2}
\end{aligned}
$$

Since $f(s(\xi)) \rightarrow f(1)$ as $\xi \rightarrow-\infty$ and the inequality (3.10) is valid for any $\varepsilon$, we have

$$
F_{1}(\xi) \leqslant i^{2}(\xi-c \tau) .
$$

Then, when $\xi \rightarrow-\infty$, we obtain

$$
e^{-2 \mu_{0} \xi} F_{1}(\xi) \leqslant e^{-2 \mu_{0} \xi} \dot{i}^{2}(\xi-c \tau) \leqslant\left[e^{-\mu_{0}(\xi-c \tau)} i(\xi-c \tau)\right]^{2} e^{-2 \mu_{0} c \tau} \leqslant\left[\sup _{\xi \in R}\left\{e^{-\mu_{0}(\xi-c \tau)} i(\xi-c \tau)\right\}\right]^{2}<\infty .
$$

Note that $\lim _{\xi \rightarrow+\infty} e^{-2 \mu_{0} \xi} F_{1}(\xi)=0$. Hence, we obtain $\sup _{\xi \in R}\left\{e^{-2 \mu_{0} \xi} F_{1}(\xi)\right\}<\infty$. Thus the right-hand integral of (3.9) is defined for $\lambda \in \mathbb{C}$ with $0<\operatorname{Re} \lambda<2 \mu_{0}$. For $c \in\left(0, c^{*}\right)$, since $\Delta(\lambda, c)>0$ for all $\lambda>0$, we get that $\mathrm{L}(\lambda)$ is defined with $\lambda \in \mathbb{C}$ with $\operatorname{Re} \lambda>0$. However, (3.9) can be rewritten as

$$
\int_{-\infty}^{+\infty} e^{-\lambda \xi}\left[\Delta(\lambda, c) i(\xi)-F_{1}(\xi)\right] d \xi=0
$$

But, $\lim _{\lambda \rightarrow+\infty} \Delta(\lambda, c)=+\infty$ for any fixed $c \in\left(0, c^{*}\right)$. This leads to a contradiction.

\section{Numerical simulation}

In this section, to further illustrate our conclusions, we perform some numeric simulations. Take

$$
\left\{\begin{array}{l}
S_{t}(x, t)=d_{1} S_{x x}(x, t)+\mu(1-S(x, t))-\frac{\beta S(x, t) I(x, t-\tau)}{1+\alpha I(x, t-\tau)} \\
I_{t}(x, t)=d_{2} I_{x x}(x, t)+\frac{\beta S(x, t) I(x, t-\tau)}{1+\alpha I(x, t-\tau)}-(\mu+\gamma) I(x, t)
\end{array}\right.
$$

where $\tau=1, \gamma=0.25, d_{1}=1, d_{2}=1, \mu=0.75, \alpha=2, \beta=4$, and the initial susceptible $S(1, x)=10$.

Let $S(x, t)=s(\xi), I(x, t)=i(\xi), \xi=x+c t$, then

$$
\left\{\begin{array}{l}
c s^{\prime}(\xi)=d_{1} s^{\prime \prime}(\xi)+\mu(1-s(\xi))-s(\xi) \frac{\beta i(\xi-c \tau)}{1+\alpha i(\xi-c \tau)} \\
c i^{\prime}(\xi)=d_{2} i^{\prime \prime}(\xi)+s(\xi) \frac{\beta i(\xi-c \tau)}{1+\alpha i(\xi-c \tau)}-(\mu+\gamma) i(\xi)
\end{array}\right.
$$

It is easy to verify that the conditions of Theorem 1.1 are satisfied. Then the traveling wave solutions of system (4.1) are presented in Figs.1 and 2. 


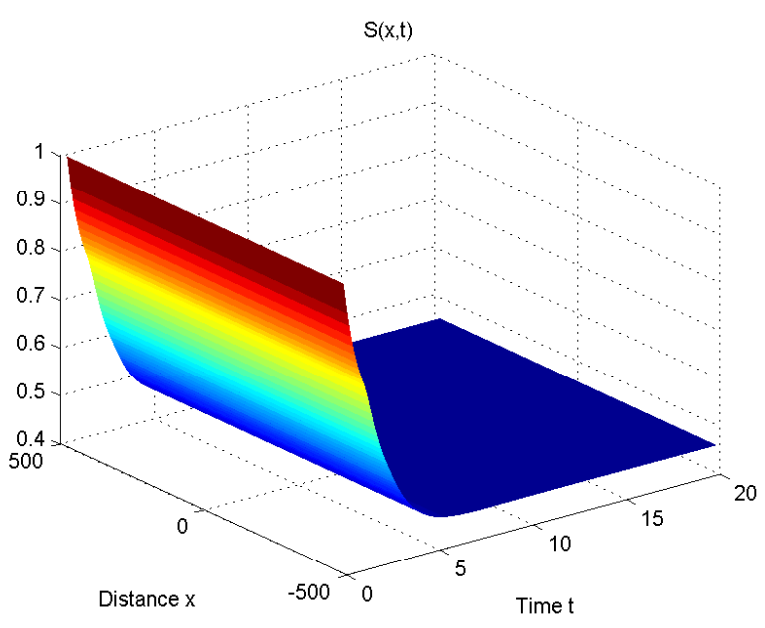

Figure 1: The traveling wave solution $S(x, t)$.

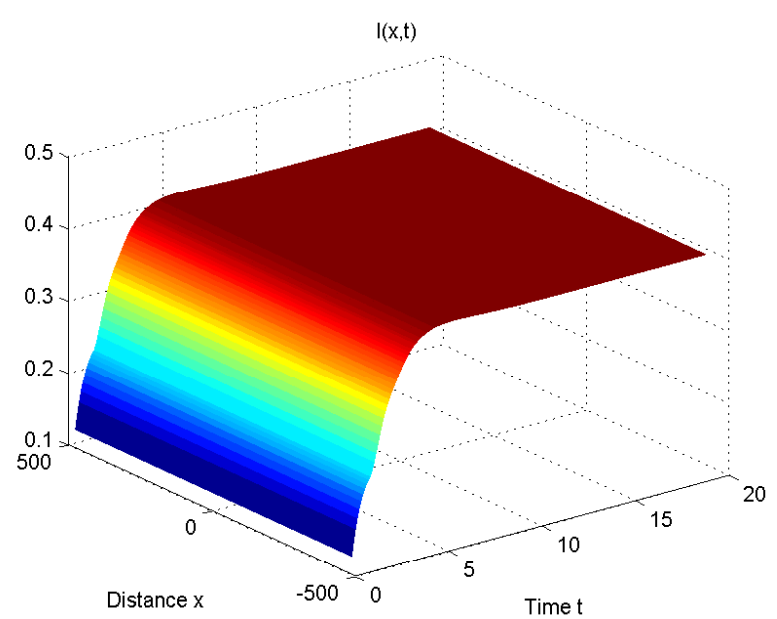

Figure 2: The traveling wave solution $I(x, t)$.

\section{Discussion}

In this paper, we consider a diffusive SIR model with delay and a general nonlinear incidence. By using Schauder's fixed point theorem and constructing a suitable Lyapunov functional, we establish the existence of traveling wave solution satisfying system (1.3). The non-existence of traveling wave solution of system (1.3) is obtained by two-sided Laplace transform. Here we prove the existence and non-existence of such a traveling wave solution is totally determined by $R_{0}$. Moreover, by Lemma 2.1, we know that $c^{*}$ is dependent on the latent period $\tau$ of disease and the diffusion rate $d_{2}$ of the infected individuals. More specifically, the minimum wave speed $c^{*}$ is determined by the following equation

$$
\begin{gathered}
\Delta(\lambda, c)=d_{2} \lambda^{2}-c \lambda+f(1) g^{\prime}(0) e^{-\lambda c \tau}-\mu-\gamma=0, \\
\frac{\partial}{\partial \lambda} \Delta(\lambda, c)=2 d_{2} \lambda-c-c \tau f(1) g^{\prime}(0) e^{-\lambda c \tau}=0 .
\end{gathered}
$$

And a direct calculation yields that

$$
\frac{\partial c^{*}}{\partial \tau}=-\frac{c^{*} f(1) g^{\prime}(0) e^{-\lambda^{*} c^{*} \tau}}{1+\tau f(1) g^{\prime}(0) e^{-\lambda^{*} c^{*} \tau}}<0, \quad \frac{\partial c^{*}}{\partial d_{2}}=\frac{\lambda^{*}}{1+\tau f(1) g^{\prime}(0) e^{-\lambda^{*} c^{*} \tau}}>0
$$

Hence the minimal wave speed $c^{*}$ is decreasing with respect to the latent period $\tau$ and increasing with respect to the diffusion rate $d_{2}$ of infected individuals.

Further, our model includes more general nonlinear incidences. In particular, if we take

$$
f(S(x, t)) g(I(x, t-\tau))=\frac{\beta S(x, t) I(x, t-\tau)}{1+\alpha I(x, t-\tau)}
$$

with $\alpha, \beta>0$, system (1.3) reduces to the model in [4]. Obviously, for any $c>c^{*}$, our results directly extend those in [4] to a more general case. In addition, for $R_{0}>1$ and $c \in\left(0, c^{*}\right)$, we prove the nonexistence of traveling wave of (1.3).

\section{Appendix}

Lemma A.1 Suppose that $\left(\mathrm{A}_{1}\right)$ and $\left(\mathrm{A}_{2}\right)$ hold and $\mathrm{R}_{0}>1$. Then system (1.3) exists a unique positive endemic equilibrium $\left(\mathrm{s}^{*}, \mathrm{i}^{*}\right)$. 
Proof. Let

$$
\mathrm{G}_{1}(\mathrm{i})=\mathrm{f}\left(1-\frac{\mu+\gamma}{\mu} \mathrm{i}\right) \mathrm{g}(\mathrm{i}), \quad \mathrm{G}_{2}(\mathrm{i})=(\mu+\gamma) \mathrm{i} .
$$

Then

$$
G_{1}^{\prime}(\mathfrak{i})=-\frac{\mu+\gamma}{\mu} f^{\prime}\left(1-\frac{\mu+\gamma}{\mu} \mathfrak{i}\right) g(\mathfrak{i})+f\left(1-\frac{\mu+\gamma}{\mu} \mathfrak{i}\right) g^{\prime}(\mathfrak{i})
$$

Note that

$$
G_{1}^{\prime}(0)=f(1) g^{\prime}(0)>\mu+\gamma, \quad G_{1}\left(\frac{\mu}{\mu+\gamma}\right)=f(0) g\left(\frac{\mu}{\mu+\gamma}\right)=0 .
$$

We obtain that $G_{1}(i)$ and $G_{2}(i)$ have at least one intersection point. Suppose that $\left(s^{*}, i^{*}\right)$ is the first positive endemic equilibrium of system (1.3). Next, we prove $\left(s^{*}, i^{*}\right)$ is a unique positive endemic equilibrium. For contradiction, we assume that $\left(s_{1}, i_{1}\right)$ is the second positive endemic equilibrium, then $G_{1}^{\prime}\left(i_{1}\right) \geqslant \mu+\gamma$. On the other hand,

$$
G_{1}^{\prime}\left(i_{1}\right)=-\frac{\mu+\gamma}{\mu} f^{\prime}\left(1-\frac{\mu+\gamma}{\mu} i_{1}\right) g\left(i_{1}\right)+f\left(1-\frac{\mu+\gamma}{\mu} i_{1}\right) g^{\prime}\left(i_{1}\right) .
$$

It follows from $\left(\mathrm{A}_{2}\right)$ that

$$
\frac{g\left(i_{1}\right)}{i_{1}}=\frac{g\left(i_{1}\right)-g(0)}{i_{1}}=g^{\prime}\left(\xi_{i_{1}}\right) \geqslant g^{\prime}\left(i_{1}\right), \quad 0<\xi_{i_{1}}<i_{1} .
$$

This, together with $f\left(1-\frac{\mu+\gamma}{\mu} i_{1}\right)=\frac{(\mu+\gamma) i_{1}}{g\left(i_{1}\right)}$, yields

$$
G_{1}^{\prime}\left(i_{1}\right)=-\frac{\mu+\gamma}{\mu} f^{\prime}\left(1-\frac{\mu+\gamma}{\mu} i_{1}\right) g\left(i_{1}\right)+(\mu+\gamma) \frac{\mathfrak{i}_{1} g^{\prime}\left(i_{1}\right)}{g\left(i_{1}\right)}<\mu+\gamma .
$$

This is a contradiction.

\section{Acknowledgment}

This work was supported by the National Natural Science Foundation of China (No. 11471197) and the National Science Foundation of Shanxi Province (No. 201601D202002).

\section{References}

[1] Z. G. Bai, S.-L. Wu, Traveling waves in a delayed SIR epidemic model with nonlinear incidence, Appl. Math. Comput., 263 (2015), 221-232. 1, 2

[2] E. Beretta, T. Hara, W. Ma, Y. Takeuchi, Global asymptotic stability of an SIR epidemic model with distributed time delay, Nonlinear Anal., 47 (2001), 4107-4115. 1

[3] J. Fang, J. J. Wei, X.-Q. Zhao, Spatial dynamics of a nonlocal and time-delayed reaction diffusion system, J. Differential Equations, 245 (2008), 2749-2770. 1

[4] S.-C. Fu, Traveling waves for a diffusive SIR model with delay, J. Math. Anal. Appl., 435 (2016), 20-37. 1, 5

[5] Y. Hosono, B. Ilyas, Traveling waves for a simple diffusive epidemic model, Math. Models Methods Appl. Sci., 5 (1995), 935-966. 1

[6] G. Huang, Y. Takeuchi, W. Ma, D. Wei, Global stability for delay SIR and SEIR epidemic models with nonlinear incidence rate, Bull. Math. Biol., 72 (2010), 1192-1207. 1

[7] W. O. Kermack, A. G. Mckendrick, A contribution to the mathematical theory of epidemics, Proc. Roy. Soc. London Ser. A, 115 (1927), 700-721. 1

[8] A. Korobeinikov, Global properties of infectious disease models with nonlinear incidence, Bull. Math. Biol., 69 (2007), 1871-1886. 1

[9] A. Korobeinikov, Lyapunov functions and global stability for SIR and SIRS epidemiological models with non-linear transmission, Bull. Math. Biol., 68 (2006), 615-626.

[10] A. Korobeinikov, P. K. Maini, Non-linear incidence and stability of infectious disease models, Math. Med. Biol., 22 (2005), 113-128. 1 
[11] W. Ma, Y. Takeuchi, T. Hara, E. Beretta, Permanence of an SIR epidemic model with distributed time delays, Tohoku Math. J. (2), 54 (2002), 581-591. 1

[12] S. G. Ruan, W. D. Wang, Dynamical behavior of an epidemic model with a nonlinear incidence rate, J. Differential Equations, 188 (2003), 135-163.

[13] M. A. Safi, A. B. Gumel, The effect of incidence functions on the dynamics of a quarantine/isolation model with time delay, Nonlinear Anal. Real World Appl., 12 (2011), 215-235.

[14] Y. Takeuchi, W. Ma, E. Beretta, Global asymptotic properties of a delay SIR epidemic model with finite incubation times, Nonlinear Anal. Ser. A: Theory Methods, 42 (2000), 931-947. 1

[15] P. X. Weng, X.-Q. Zhao, Spreading speed and traveling waves for a multi-type SIS epidemic model, J. Differential Equations, 229 (2006), 270-296. 1

[16] C. F. Wu, P. X. Weng, Asymptotic speed of propagation and traveling wavefronts for a SIR epidemic model, Discrete Contin. Dyn. Syst. Ser. B, 15 (2011), 867-892. 1

[17] Z. Xu, Traveling waves in a Kermack-Mckendrick epidemic model with diffusion and latent period, Nonlinear Anal., 111 (2014), 66-81. 1

[18] R. Xu, Z. Ma, Global stability of a SIR epidemic model with nonlinear incidence rate and time delay, Nonlinear Anal. Real World Appl., 10 (2009), 3175-3189. 1 